\title{
Non-perturbative Correlation Effects in Diluted Magnetic Semiconductors
}

\author{
M. Cygorek, ${ }^{1}$ P. I. Tamborenea, ${ }^{2,1}$ and V. M. Axt ${ }^{1}$ \\ ${ }^{1}$ Theoretische Physik III, Universität Bayreuth, 95440 Bayreuth, Germany \\ ${ }^{2}$ Departamento de Física and IFIBA, FCEN, Universidad de Buenos Aires, \\ Ciudad Universitaria, Pabellón I, 1428 Ciudad de Buenos Aires, Argentina
}

\begin{abstract}
The effects of carrier-impurity correlations due to a Kondo-like spin-spin interaction in diluted magnetic semiconductors are investigated. These correlations are not only responsible for a transfer of spins between the carriers and the impurities, but also produce non-perturbative effects in the spin dynamics such as renormalization of the precession frequency of the carrier spins, which can reach values of several percent in CdMnTe quantum wells. In two-dimensional systems, the precession frequency renormalization for a single electron spin with defined wave vector shows logarithmic divergences similar to those also known from the Kondo problem in metals. For smooth electron distributions, however, the divergences disappear due to the integrability of the logarithm. A possible dephasing mechanism caused by the wave-vector dependence of the electron spin precession frequencies is found to be of minor importance compared to the spin transfer from the carrier to the impurity system. In the Markov limit of the theory, a quasi-equilibrium expression for the carrier-impurity correlation energy can be deduced indicating the formation of strongly correlated carrier-impurity states for temperatures in the $\mathrm{mK}$ range.
\end{abstract}

PACS numbers: 75.78.Jp, 75.50.Pp, 75.30.Hx, 72.10.Fk

\section{INTRODUCTION}

A perturbative treatment of the interaction between quasi-free electrons in a metal with localized magnetic impurities predicts logarithmic divergences in several quantities such as resistivity and entropy at zero temperature ${ }^{1,2}$. This finding, the Kondo effect, is a famous example of a situation where perturbation theory leads to unphysical conclusions whereas the measured values of the resistivity assume finite values. The Kondo problem, i. e., the question of how to properly describe the low-temperature limit of a system with a spin dependent carrier-impurity interaction theoretically, has opened up a wide field of phyiscs. Although the Kondo problem, as it was originally formulated, has been solved $^{2}$, the Kondo physics experienced a revival since it has become possible to study experimentally similar problems in other systems, e. g., structures where quantum dots play the role of the magnetic impurities ${ }^{3-12}$. The common feature in these systems is that a microscopic exchange coupling gives rise to an effective KondoHamiltonian that assumes the form of a spin-spin contact interaction between the quasi-free carriers and the localized magnetic impurities, or quantum dots, respectively.

Other systems which are usually modelled by a Kondolike Hamiltonian are diluted magnetic semiconductors (DMS) where typically II-VI or III-V semiconductors are doped with magnetic impurities, usually $\mathrm{Mn}$ which effectively forms a spin- $\frac{5}{2}$ system. These materials have been studied extensively in the last decades ${ }^{13-33}$ due to their optical and magnetic properities which make them promising candidates for future spintronics devices ${ }^{34-36}$. While the carrier-impurity correlations play a crucial role in the metallic Kondo problem, they are often neglected in studies about DMS by employing a mean-field approximation $^{24}$. In some articles ${ }^{37}$ it is argued that the situation in DMS is different from the original Kondo situation in that in the latter only a few magnetic impurites and a huge number of quasi-free carriers are present in the metal, whereas in the former case, in particular in the case of (intrinsic) II-VI DMS, the number of impurities usually exceeds the number of carriers.

On the other hand, a third-order many-body perturbation theory based on the pseudofermion formalism ${ }^{38}$ reveals Kondo-like divergences in the propagator for the spin dynamics in DMS due to the hole-impurity exchange interaction. From this it was concluded that the carrierimpurity correlations should in fact be important for the dynamics in DMS. Furthermore, some studies ${ }^{39-44}$ suggest that bound magnetic polarons can play a key role for the ferromagnetic behaviour of some DMS. These polarons consist of carriers which are bound to the magnetic impurities and, thus, the carriers and impurities are strongly correlated.

In this article, we address the question of the importance of carrier-impurity correlations in DMS and the relevance of possible Kondo-like divergences. We base our study on a microscopic quantum kinetic theory derived by a correlation expansion scheme ${ }^{45}$ that is capable of a non-perturbative description of highly nonequilibrium situations. One aspect of the effects of the carrier-impurity correlations on the spin dynamics has already been found in previous works ${ }^{46-50}$ : The correlations mediate the transfer of spins between the carriers and the impurities. Since in the Markovian limit, the quantum kinetic theory contains the special case of rate equations which can also be derived by a Fermi's golden rule approach ${ }^{46}$, this spin transfer can, in fact, be treated perturbatively ${ }^{51}$. Note that in some situations, e. g., for excitations close to the band edge in two- and lower-dimensional DMS ${ }^{52}$, the Markov limit is not a good approximation so that deviations from a golden-rule-like 
exponential decay are predicted.

In the present study, we show that the carrier-impurity correlations are also responsible for another effect in the spin dynamics that is not predicted by a perturbative method: a renormalization of the precession frequency of carrier spins compared with its mean-field value. It is shown that the frequency renormalization also contains Kondo-like logarithmic divergences in the Markov limit in two-dimensional systems. However, these divergences never lead to unphysical results in the spin dynamics. This is, first of all, due to the fact that the singularities are integrable and yield finite values for a non-singular spectral electron distribution. Moreover, the divergence in the frequency renormalization is only found for $t \rightarrow \infty$ where the amplitude of the precessing electron spin has already decayed to zero. The Markov limit of the quantum kinetic theory also allows to find an expression for the carrier-impurity correlation energy which shows a similar behaviour as the frequency renormalization, including Kondo-like logarithmic divergences in the twodimensional case.

The article is structured as follows: First, the quantum kinetic theory is briefly reviewed as well as effective (PESC, precession of electrons and correlations) equations ${ }^{49}$ based on the quantum kinetic theory. Then, the frequency renormalization described by the PESC equations is calculated and compared with the result of a Markovian approximation to the PESC equations in two and three dimensions. A possible electron spin dephasing mechanism due to the wave vector dependence of the frequency renormalization is discussed. Finally, we investigate the mean carrier-impurity correlation energy.

\section{THEORY}

\section{A. System}

The Hamiltonian for conduction band electrons in DMS is modelled by

$$
\begin{aligned}
& H=H_{0}+H_{s d} \\
& H_{0}=\sum_{\mathbf{k} \sigma} \hbar \omega_{\mathbf{k}} c_{\sigma \mathbf{k}}^{\dagger} c_{\sigma \mathbf{k}} \\
& H_{s d}=\frac{J_{s d}}{V} \sum_{I n n^{\prime} \mathbf{k} \mathbf{k}^{\prime} \sigma \sigma^{\prime}} \mathbf{S}_{n n^{\prime}} \cdot \mathbf{s}_{\sigma \sigma^{\prime}} c_{\sigma \mathbf{k}}^{\dagger} c_{\sigma^{\prime} \mathbf{k}^{\prime}} e^{i\left(\mathbf{k}^{\prime}-\mathbf{k}\right) \mathbf{R}_{I}} \hat{P}_{n n^{\prime}}^{I}
\end{aligned}
$$

where $H_{0}$ describes the band structure and $H_{s d}$ is the Kondo Hamiltonian which originates from the exchange interaction between the $s$-type conduction band electrons and the $d$-electrons of the magnetic ions. Throughout this article, we assume a parabolic band structure with $\omega_{\mathbf{k}}=\frac{\hbar k^{2}}{2 m^{*}}$ where $m^{*}$ is the effective mass. $J_{s d}$ and $V$ are the coupling constant and volume of the DMS, $c_{\sigma \mathbf{k}}^{\dagger}$ and $c_{\sigma \mathbf{k}}$ are the creation and annihilation operators for electrons with spin index $\sigma$ and wave vector $\mathbf{k}$. $\mathbf{R}_{I}$ is the position of the $I$-th magnetic impurity and $\hat{P}_{n n^{\prime}}^{I}=$ $|I, n\rangle\left\langle I, n^{\prime}\right|$ are the projection operators corresponding to the spin state of the $I$-th impurity, e. g., for spin $\frac{5}{2} \mathrm{Mn}$ impurities, $n=\left\{-\frac{5}{2},-\frac{3}{2} \ldots \frac{5}{2}\right\} . \mathbf{S}_{n n^{\prime}}$ and $\mathbf{s}_{\sigma \sigma^{\prime}}$ are the spin matrices for spin $\frac{5}{2}$ and $\frac{1}{2}$ systems, respectively.

\section{B. Equations of motion}

A microscopic quantum kinetic theory based on a correlation expansion scheme was constructed in Ref. 45, where equations of motion have been derived for the electron and impurity density matrices $C_{\sigma_{1} \mathbf{k}}^{\sigma_{2}}$ and $M_{n_{1}}^{n_{2}}$ as well as their correlations which are defined by

$$
\begin{aligned}
C_{\sigma_{1} \mathbf{k}}^{\sigma_{2}}= & \left\langle c_{\sigma_{1} \mathbf{k}}^{\dagger} c_{\sigma_{2} \mathbf{k}}\right\rangle \\
M_{n_{1}}^{n_{2}}= & \left\langle\hat{P}_{n_{1} n_{2}}^{I}\right\rangle \\
Q_{\sigma_{1} n_{1} \mathbf{k}_{1}}^{\sigma_{2} n_{2}}= & V\left(\left\langle c_{\sigma_{1} \mathbf{k}_{1}}^{\dagger} c_{\sigma_{2} \mathbf{k}_{2}} e^{i\left(\mathbf{k}_{2}-\mathbf{k}_{1}\right) \mathbf{R}_{I}} \hat{P}_{n_{1} n_{2}}^{I}\right\rangle+\right. \\
& \left.-\left\langle c_{\sigma_{1} \mathbf{k}_{1}}^{\dagger} c_{\sigma_{2} \mathbf{k}_{2}} e^{i\left(\mathbf{k}_{2}-\mathbf{k}_{1}\right) \mathbf{R}_{I}}\right\rangle\left\langle\hat{P}_{n_{1} n_{2}}^{I}\right\rangle\right)
\end{aligned}
$$

where the brackets denote the quantum mechanical average as well as an average over homogeneously distributed impurities. The equations of motion for these dynamical variables are given in Ref. 48.

The full quantum kinetic equations are lengthy and their solution requires considerable numerical effort. However, it was found in Ref. 49 that they can be drastically simplified in the case where the number of impurity ions $N_{M n}$ is much larger than the number of the quasi-free electrons $N_{e}$. This is usually fulfilled especially in II-VI DMS where the magnetic doping with Mn does not simultaneously lead to p- or n-doping and the carriers stem exclusively from optical excitation. To understand the effective equations derived in Ref. 49 it is instructive to first consider the mean-field dynamics for the spin $\mathbf{s}_{\mathbf{k}}=\sum_{\sigma_{1} \sigma_{2}} \mathbf{s}_{\sigma_{1} \sigma_{2}} C_{\sigma_{1} \mathbf{k}}^{\sigma_{2}}$ of electrons with wave vector $\mathbf{k}$ and the impurities $\langle\mathbf{S}\rangle=\sum_{n_{1} n_{2}} \mathbf{S}_{n_{1} n_{2}} M_{n_{1}}^{n_{2}}$. In the mean-field approximation, i. e., if the correlations are neglected, one finds

$$
\begin{aligned}
& \left.\frac{\partial}{\partial t} \mathbf{s}_{\mathbf{k}}\right|_{M F}=\boldsymbol{\omega}_{M} \times \mathbf{s}_{\mathbf{k}}, \\
& \left.\frac{\partial}{\partial t}\langle\mathbf{S}\rangle\right|_{M F}=-\left.\frac{1}{N_{M n}} \sum_{\mathbf{k}} \frac{\partial}{\partial t} \mathbf{s}_{\mathbf{k}}\right|_{M F},
\end{aligned}
$$

where $\boldsymbol{\omega}_{M}:=\frac{J_{s d}}{\hbar} n_{M n}\langle\mathbf{S}\rangle$. Eq. (3b) follows from the total spin conservation of the Kondo Hamiltonian. In the case $N_{M n} \gg N_{e}$, the change of the impurity spin is marginal and can therefore be neglected. The precession of the electron spin around the mean field due to the impurity magnetization, on the other hand, is in general important. Eq. (3a) is solved by

$$
\mathbf{s}_{\mathbf{k}}=R_{\langle\mathbf{S}\rangle}\left(\omega_{M} t\right) \mathbf{s}_{\mathbf{k}}^{\prime},
$$

where $R_{\mathbf{n}}(\alpha)$ is the matrix describing a rotation around the vector $\mathbf{n}$ with angle $\alpha$ and the precession frequency 
$\omega_{M}=\boldsymbol{\omega}_{M} \cdot\langle\mathbf{S}\rangle /|\langle\mathbf{S}\rangle|$ is defined so that it has the same sign as the coupling constant $J_{s d}$. In the mean field approximation $\mathbf{s}_{\mathbf{k}}^{\prime}$ is constant. However, if we also account for the carrier-impurity correlations, $\mathbf{s}_{\mathbf{k}}^{\prime}$ changes slowly with time and constitutes the electron spin in a rotating frame. If the correlations are formally integrated and inserted into the corresponding equations of motion for the electron variables, the effective equations for the electron spin component $\mathbf{s}^{\prime} \mathbf{k}_{1}$ perpendicular to the impurity magnetization can be given as $^{49}$ :

$$
\begin{aligned}
\frac{\partial}{\partial t} \mathbf{s}^{\prime \perp}{ }_{\mathbf{k}_{1}}^{\perp}= & -\sum_{\mathbf{k}}\left[\operatorname{Re}\left(G_{\omega_{\mathbf{k}}}^{\omega_{\mathbf{k}_{1}}-\omega_{M}}\right)\left(\frac{b^{+}}{2}-b^{0} n_{\mathbf{k}}^{\uparrow}\right) \mathbf{s}_{\mathbf{k}_{1}}^{\prime \perp}+\right. \\
& +\operatorname{Re}\left(G_{\omega_{\mathbf{k}_{1}}}^{\omega_{\mathbf{k}_{1}}+\omega_{M}}\right)\left(\frac{b^{-}}{2}+b^{0} n_{\mathbf{k}}^{\downarrow}\right) \mathbf{s}_{\mathbf{k}_{1}}^{\prime \perp}+ \\
& \left.+\operatorname{Re}\left(G_{\omega_{\mathbf{k}}}^{\omega_{\mathbf{k}_{1}}}\right) \frac{b^{\|}}{2}\left(\mathbf{s}_{\mathbf{k}}^{\prime}+\mathbf{s}_{\mathbf{k}_{1}}^{\prime \perp}\right)\right]+ \\
& -\frac{\langle\mathbf{S}\rangle}{|\langle\mathbf{S}\rangle|} \times \sum_{\mathbf{k}}\left[\operatorname{Im}\left(G_{\omega_{\mathbf{k}}}^{\omega_{\mathbf{k}_{1}}-\omega_{M}}\right)\left(\frac{b^{+}}{2}-b^{0} n_{\mathbf{k}}^{\uparrow}\right)+\right. \\
& \left.-\operatorname{Im}\left(G_{\omega_{\mathbf{k}}}^{\omega_{\mathbf{k}_{1}}+\omega_{M}}\right)\left(\frac{b^{-}}{2}+b^{0} n_{\mathbf{k}}^{\downarrow}\right)\right] \mathbf{s}_{\mathbf{k}_{1}}^{\prime \perp},
\end{aligned}
$$

The coefficients in Eq. (5) are given by $b^{ \pm}:=\left\langle S^{\perp^{2}}\right\rangle \pm$ $\frac{\left\langle S^{\|}\right\rangle}{2}, b^{0}:=\frac{\left\langle S^{\|}\right\rangle}{2}$ and $b^{\|}:=\left\langle S^{\|^{2}}\right\rangle$, where the component of the impurity spin operator in the direction of the mean impurity spin is $S^{\|}:=\hat{\mathbf{S}} \cdot \frac{\langle\hat{\mathbf{S}}\rangle}{|\langle\hat{\mathbf{S}}\rangle|}$, and the relevant second moments of the impurity spin operator can be separated into parallel $\left\langle S^{\|^{2}}\right\rangle$ and perpendicular parts $\left\langle S^{\perp^{2}}\right\rangle=\frac{1}{2}\left\langle S^{2}-S^{\|^{2}}\right\rangle$. The memory function

$$
G_{\omega_{\mathbf{k}}}^{\omega_{\mathbf{k}_{1}}}:=\frac{J_{s d}^{2}}{\hbar^{2}} \frac{n_{M n}}{V} \int_{-t}^{0} d t^{\prime} e^{i\left(\omega_{\mathbf{k}}-\omega_{\mathbf{k}_{1}}\right) t^{\prime}}
$$

has to be interpreted as an integral operator and the time-dependent variables that appear after $G_{\omega_{\mathbf{k}}}^{\omega_{\mathbf{k}_{1}}}$ in Eq. (5) are evaluated at $t^{\prime}$. Finally, $n_{\mathbf{k}}^{\uparrow / \downarrow}$ are the occupation numbers of the states with wave-vector $\mathbf{k}$, i. e., the diagonal elements of the density matrix with respect to the spin indices. Eq. (5) together with the corresponding equations for $n_{\mathbf{k}}^{\uparrow / \downarrow}$ given in Ref. 49 are called precession of electron spins and correlations (PESC) equations, since besides the electron spin, also the correlations $Q_{\beta \mathbf{k}_{1}}^{\alpha \mathbf{k}_{2}}:=\sum_{\sigma_{1} \sigma_{2}} \sum_{n_{1} n_{2}} \mathbf{s}_{\sigma_{1} \sigma_{2}} \cdot \mathbf{S}_{n_{1} n_{2}} Q_{\sigma_{1} n_{1} \mathbf{k}_{1}}^{\sigma_{2} n_{2} \mathbf{k}_{2}}$ exhibit a precession-like movement around the mean field due to the impurity magnetization. Note that Eq. (5) is equivalent to the full quantum kinetic theory of Ref. 45 except that some source terms for the correlations are neglected that are numerically insignificant [cf. Ref. 49 for details].

Eq. (5) is only complicated and numerically challenging due to the time integral induced by the memory function $G_{\omega_{\mathbf{k}}}^{\omega_{\mathbf{k}_{1}}}$. Now, working in the rotating frame allows us to assume that the electron variables change only slowly in time and can equally well be evaluated at $t$ instead of $t^{\prime}$. The memory integral consists then only of

$$
\int_{-t}^{0} d t^{\prime} e^{i\left(\omega_{\mathbf{k}}-\omega_{\mathbf{k}_{1}}\right) t^{\prime}} \approx \pi \delta\left(\omega_{\mathbf{k}}-\omega_{\mathbf{k}_{1}}\right)-i \mathcal{P} \frac{1}{\omega_{\mathbf{k}}-\omega_{\mathbf{k}_{1}}},
$$

where $\mathcal{P}$ is the Cauchy principal value. The Markov approximation (7) was established by letting $t \rightarrow \infty$ in the lower limit of the integral and using the SokhotskiPlemelj theorem. The validity of the Markovian approximation can in general depend on the values of $\mathbf{k}, \mathbf{k}_{1}$, $t$ as well as the timescale of the change of the electron variables and therefore has to be checked numerically.

If only the real part of the memory function is used in Markov approximation and the imaginary part is neglected, the PESC-equations assume a golden rule-type form, where the spin transfer dynamics follows approximately an exponential decay to the equilibrium value with rate

$$
\begin{aligned}
\left(\tau_{\perp}\right)^{-1} \approx & \frac{J_{s d}^{2} n_{M n}}{\hbar^{2} V} \pi\left[D\left(\omega_{1}-\omega_{M}\right) \frac{b^{+}}{2}+D\left(\omega_{1}+\omega_{M}\right) \frac{b^{-}}{2}+\right. \\
& \left.+D\left(\omega_{1}\right) b^{\|}\right]
\end{aligned}
$$

for an electron with kinetic energy $\omega_{1}$, if the terms of second order of the electron variables in Eq. (5) are neglected $^{49}$. In the expression for the rate, $D(\omega)$ describes the spectral density of states and depends on the dimensionality of the system.

\section{Frequency renormalization in the Markov limit}

One issue that we would like to focus on in the present work is the change in the precession frequency described in Eq. (5) by the terms proportional to the imaginary part of the memory function. Such a renormalization of the precession frequency would be absent in any truncated perturbative approach ${ }^{53}$. It originates, like the spin transfer described by the real part of the memory function, from the carrier-impurity correlations.

It is noteworthy that the frequency renormalization is singular in the Markov limit described in Eq. (7), i. e., the imaginary part of the memory function $G_{\omega_{\mathbf{k}}}^{\omega_{\mathbf{k}_{1}}}$ diverges if $\omega_{\mathbf{k}}=\omega_{\mathbf{k}_{1}}$. However, this divergence does not lead to an unphysical behaviour. First of all, the divergence is a feature of the Markovian limit. For finite times $t$, the l. h. s. of Eq. (7) is a finite integral over an analytic function and is therefore also analytic. For $\omega_{\mathbf{k}}=\omega_{\mathbf{k}_{1}}$, the value of the integral is $t$ which only goes to infinity in the Markov limit. As only the electron spin component perpendicular to the impurity magnetization is affected by the frequency renormalization and this component decays approximately exponentially to zero, an infinite precession frequency is never observable.

Similar to the Markovian spin transfer rate in Eq. (8), an expression for the frequency renormalization $\Delta \omega$ can be given in the Markov limit of Eq. (5), if the imaginary part of Eq. (7) is used: 


$$
\begin{aligned}
& \Delta \omega\left(\omega_{1}\right)=\frac{J_{s d}^{2}}{\hbar^{2}} \frac{n_{M n}}{V} \int_{0}^{\omega_{B} Z} d \omega D(\omega) \times \\
& {\left[\frac{b^{+}}{2} \frac{1}{\omega-\left(\omega_{1}-\omega_{M}\right)}-\frac{b^{-}}{2} \frac{1}{\omega-\left(\omega_{1}+\omega_{M}\right)}\right],}
\end{aligned}
$$

where, for the sake of simplicity, the terms proportional to $n^{\uparrow / \downarrow}$ in Eq. (5) were neglected, since they only matter if a large number of carriers is present. In two-dimensional systems, the spectral density of states $D^{2 d}(\omega)=\frac{A m^{*}}{2 \pi \hbar} \Theta(\omega)$ is constant, where $A$ is the sample area and $\Theta(x)$ is the step function. In three dimensions, $D^{3 d}(\omega)=\frac{V}{4 \pi^{2}}\left(\frac{2 m^{*}}{\hbar}\right)^{3 / 2} \sqrt{\omega} \Theta(\omega)$ is proportional to the square-root of $\omega$. The corresponding frequency renormalizations are:

$$
\begin{aligned}
\Delta \omega^{2 d}\left(\omega_{1}\right)= & -\frac{J_{s d}^{2}}{\hbar^{2}} \frac{n_{M n}}{d} \frac{m^{*}}{2 \pi \hbar}\left\{\frac{b^{+}}{2} \ln \left|\frac{\omega_{1}-\omega_{M}}{\omega_{B Z}-\left(\omega_{1}-\omega_{M}\right)}\right|+\right. \\
& \left.-\frac{b^{-}}{2} \ln \left|\frac{\omega_{1}+\omega_{M}}{\omega_{B Z}-\left(\omega_{1}+\omega_{M}\right)}\right|\right\}
\end{aligned}
$$

where $d=V / A$ is the quantum well width, and

$$
\begin{aligned}
\Delta \omega^{3 d}\left(\omega_{1}\right)= & \frac{J_{s d}^{2}}{\hbar^{2}} \frac{n_{M n}}{4 \pi}\left(\frac{2 m^{*}}{\hbar}\right)^{3 / 2} \int_{0}^{\omega_{B Z}} d \omega \times \\
& \left\{\frac{b^{+}}{2} \frac{\sqrt{\omega}}{\omega-\left(\omega_{1}-\omega_{M}\right)}-\frac{b^{-}}{2} \frac{\sqrt{\omega}}{\omega-\left(\omega_{1}+\omega_{M}\right)}\right\},
\end{aligned}
$$

with

$$
\begin{aligned}
& \int_{0}^{\omega_{B Z}} d \omega \frac{\sqrt{\omega}}{\omega-\omega_{0}}= \\
& = \begin{cases}2 \sqrt{\omega_{B Z}}-\sqrt{\omega_{0}} \ln \mid \frac{\omega_{0}+\omega_{B Z} \mid}{\omega_{0}-\omega_{B Z} \mid,} & \omega_{0}>0 \\
2 \sqrt{\omega_{B Z}}-2 \sqrt{\left|\omega_{0}\right|} \tan ^{-1}\left(\frac{\omega_{B Z}}{\left|\omega_{0}\right|}\right), & \omega_{0}<0\end{cases}
\end{aligned}
$$

It should be noted that in two and three dimensions the frequency renormalization depends explicitly on the frequency $\omega_{B Z}$, which corresponds to the energy at the end of the first Brillouin zone, and diverges in the limit $\omega_{B Z} \rightarrow \infty$. For typical pump-probe experiments with diluted magnetic semiconductors, carriers are optically excited relatively close to the band edge. For the excited electrons, one can safely assume $\omega_{1} \pm \omega_{M} \ll \omega_{B Z}$. In this case, we find a logarithmic dependence on $\omega_{B Z}$ in the two-dimensional frequency renormalization.

With the same assumption also the integral in Eq. (10c) for the three-dimensional renormalization can be simplified to

$$
\int_{0}^{\omega_{B Z}} d \omega \frac{\sqrt{\omega}}{\omega-\omega_{0}} \approx 2 \sqrt{\omega_{B Z}}-\pi \sqrt{\left|\omega_{0}\right|} \Theta\left(-\omega_{0}\right) .
$$

Thus, we find a square-root dependence of the frequency renormalization on the cut-off frequency $\omega_{B Z}$, as well as a square-root dependence on $\omega_{0}=\omega_{1} \pm \omega_{M}$ which only contributes if $\omega_{0}$ is negative.

The divergence in the limit $\omega_{B Z} \rightarrow \infty$ is similar to the metallic Kondo effect where the divergence in the resistivity is also logarithmic in the bandwidth ${ }^{2}$. The Kondo problem in metals resembles rather the two-dimensional than the three-dimensional case in DMS, because the formalism for the solution of the Kondo problem usually describes the carrier system as possessing a constant spectral density of states ${ }^{2}$.

It is noteworthy that the divergence of the frequency renormalization for $\omega_{0}=\omega_{1} \pm \omega_{M}$ vanishes in the threedimensional case due to the integral over the density of states. In two-dimensional systems, a diverging frequency renormalization remains, but only for electrons with a unique value of the kinetic energy. For realistic optical excitation, however, a smooth spectral electron distribution can be expected so that the change of the total precession frequency comprises an averaging over frequency renormalizations of nearby states. Since the logarithmic divergence is integrable, the total frequency renormalization remains finite.

\section{NUMERICAL CALCULATIONS}

In order to check the validity of the Markov approximation for the renormalization of the precession frequency of the electrons, we compare the Markov result with calculations, where the memory is taken into account explicitly. It seems straightforward to use Eq. (5) with the time-integral operator $G_{\omega_{\mathbf{k}}}^{\omega_{\mathbf{k}_{1}}}$ defined in Eq. (6) and solve the integro-differential equations numerically. This is, however, a very challenging problem for the following reasons.

From the Markovian expression for the frequency renormalization, we find the explicit dependence on the value of the cut-off energy $\hbar \omega_{B Z}$. Therefore, also oscillations with frequencies close to $\omega_{B Z}$ have to be resolved, which are on the timescale of a few fs since $\hbar \omega_{B Z}$ is in the $\mathrm{eV}$ range. On the other hand, relevant changes of the total electron spin takes place in the 10-100 ps range. Furthermore, for each time step the calculation of each $\mathbf{s}_{\mathbf{k}_{1}}^{\prime \perp}$ requires a sum over all possible $\mathbf{k}$-states so that the problem has the complexity $\mathcal{O}\left(N_{k}^{2}\right)$ where $N_{k}$ is the number of discretization points for the $\mathrm{k}$-space. Note that also in $k$-space, the details of excitations close to the band edge in the $\mathrm{meV}$ range as well as the full Brillouin zone up to energies of a few eV have to be resolved. Such a problem also arises in the metallic Kondo effect where numerical procedures, such as the famous renormalization group ${ }^{54}$, have been developed to deal with the large value of the band width ${ }^{2}$. Note that solving the integrodifferential equation by finding an auxiliary variable, so that the problem can be transformed into an ordinary differential equation, is equivalent to using the original 
quantum kinetic theory ${ }^{49}$.

Here, we solve this problem by using approximations that allow a separation of electron spins with different wave vectors, so that we find a $\mathcal{O}\left(N_{k}\right)$ problem for an individual electron with wave vector $\mathbf{k}_{1}$. First of all, it is noteworthy that $\mathbf{s}_{\mathbf{k}_{1}}^{\prime \perp}$ in Eq. (5) couples to the occupations $n_{\mathbf{k}}^{\uparrow / \downarrow}$ of states with different wave vectors $\mathbf{k}$. These terms, however, are of second order in electron variables and have a marginal effect on the dynamics of the perpendicular spin component ${ }^{49}$, especially if the electron density is small, as is usually the case for optically excited carriers. Neglecting these terms, we can formulate equations of motion for the complex perpendicular electron spin variable (in the rotating frame):

$$
s_{\mathbf{k}_{1}}^{\prime}:=s_{\mathbf{k}_{1}}^{\prime x}+i s_{\mathbf{k}_{1}}^{\prime y},
$$

where it is assumed that the impurity magnetization points in the z-direction. Then, the PESC-equations (5) assume the form:

$$
\begin{aligned}
& \frac{\partial}{\partial t} s_{\mathbf{k}_{1}}^{\prime}(t)=-\frac{J_{s d}^{2}}{\hbar^{2}} \frac{n_{M n}}{V} \sum_{\mathbf{k}} \int_{0}^{t} d t^{\prime} \times \\
& \left\{\frac{b^{+}}{2} e^{i\left[\omega_{\mathbf{k}}-\left(\omega_{\mathbf{k}_{1}}-\omega_{M}\right)\right]\left(t^{\prime}-t\right)} s_{\mathbf{k}_{1}}^{\prime}\left(t^{\prime}\right)+\right. \\
& +\frac{b^{-}}{2} e^{-i\left[\omega_{\mathbf{k}}-\left(\omega_{\mathbf{k}_{1}}+\omega_{M}\right)\right]\left(t^{\prime}-t\right)} s_{\mathbf{k}_{1}}^{\prime}\left(t^{\prime}\right)+ \\
& \left.+\frac{b^{\|}}{2} \cos \left[\left(\omega_{\mathbf{k}}-\omega_{\mathbf{k}_{1}}\right)\left(t^{\prime}-t\right)\right]\left(s_{\mathbf{k}}^{\prime}\left(t^{\prime}\right)+s_{\mathbf{k}_{1}}^{\prime}\left(t^{\prime}\right)\right)\right\}
\end{aligned}
$$

It can be seen immediately from Eq. (13) that in the equation for $s_{\mathbf{k}_{1}}^{\prime}$, electron variables of states with other wave vectors only enter in the last term, i. e., the term proportional to $s_{\mathbf{k}}^{\prime}\left(t^{\prime}\right)+s_{\mathbf{k}_{1}}^{\prime}\left(t^{\prime}\right)$. Note that a time integration of $\cos \left[\left(\omega_{\mathbf{k}}-\omega_{\mathbf{k}_{1}}\right)\left(t^{\prime}-t\right)\right]$ yields $\frac{\sin \left[\left(\omega_{\mathbf{k}}-\omega_{\mathbf{k}_{1}}\right) t\right]}{\left(\omega_{\mathbf{k}}-\omega_{\mathbf{k}_{1}}\right) t}$ which has a pronounced peak at $\omega_{\mathbf{k}}=\omega_{\mathbf{k}_{1}}$. Thus, if the electron spin distribution is assumed to be a smooth function in $\mathrm{k}$ space, the main contribution of the last term in Eq. (13) will be approximately the same if we set

$$
s_{\mathbf{k}}^{\prime}\left(t^{\prime}\right) \approx s_{\mathbf{k}_{1}}^{\prime}\left(t^{\prime}\right)
$$

This approximation was shown to reproduce the nonMarkovian features of the spin transfer in Ref. 52. Also, in contrast to the other terms, the last term of Eq. (13), where the approximation is used, does not influence the frequency renormalization, due to the absence of an imaginary part of the oscillating prefactor $\cos \left[\left(\omega_{\mathbf{k}}-\omega_{\mathbf{k}_{1}}\right)\left(t^{\prime}-\right.\right.$ $t)$. Now, with the help of approximation (14), we end up with completely decoupled equations for the spins $s_{\mathbf{k}_{1}}^{\prime}$ of electrons with different wave vectors $\mathbf{k}_{1}$.

Finally, it is useful for the numerical solution of the integro-differential equation (13) to transform it into an ordinary differential equation using auxiliary variables
$G_{\mathbf{k}_{1} \mathbf{k}}^{j}:$

$$
\begin{aligned}
& \frac{\partial}{\partial t} s_{\mathbf{k}_{1}}^{\prime}=-\frac{J_{s d}^{2}}{\hbar^{2}} \frac{n_{M n}}{V} \sum_{j=1}^{4} \sum_{\mathbf{k}} D(\mathbf{k}) G_{\mathbf{k}_{1} \mathbf{k}}^{j} \\
& \frac{\partial}{\partial t} G_{\mathbf{k}_{1} \mathbf{k}}^{j}=\sigma_{j} i\left(\omega_{\mathbf{k}}-\omega_{\mathbf{k}_{1}}+\chi_{j} \omega_{M}\right) G_{\mathbf{k}_{1} \mathbf{k}}^{j}+\frac{b_{j}}{2} s_{\mathbf{k}_{1}}^{\prime},
\end{aligned}
$$

with

$$
\begin{aligned}
& \sigma_{j}=\{1,-1,1,-1\}, \\
& \chi_{j}=\{1,-1,0,0\}, \\
& b_{j}=\left\{b^{+}, b^{-}, b^{\|}, b^{\|}\right\}
\end{aligned}
$$

and initial conditions $G_{\mathbf{k}_{1} \mathbf{k}}^{j}=0$ for $t=0$. Calculating the dynamics of a single electron spin using Eqs. (15) has the complexity $\mathcal{O}\left(N_{k}\right)$ and can be done without the need for a numerical renormalization group procedure.

\section{RESULTS FOR THE FREQUENCY RENORMALIZATION}

The parameters used for the numerical calculations describe a $\mathrm{Cd}_{0.93} \mathrm{Mn}_{0.07} \mathrm{Te}$ sample with coupling constant $J_{s d}=-15 \mathrm{meVnm}^{3}$, effective mass $m^{*}=0.093 m_{0}{ }^{55}$, where $m_{0}$ is the free electron mass, and, in the case of a two-dimensional system, a quantum well width of $d=5$ $\mathrm{nm}$. The cut-off energy was taken to be $\hbar \omega_{B Z}=3 \mathrm{eV}$. The initial impurity magnetization was modelled to be thermally distributed and is therefore completely defined by the mean value $\left\langle S^{\|}\right\rangle \in\left[-\frac{5}{2} ; \frac{5}{2}\right]$.

We assume that electrons have been spin selectively prepared by optical excitation with circularly polarized light so that the initial electron spin is perpendicular to the initial impurity magnetization (Voigt geometry). Eqs. (15) are used to calculate the finite-memory spin dynamics for electrons with a defined wave vector $k_{1}$ or, equivalently, kinetic energy $\hbar \omega_{1}=\frac{\hbar^{2} k_{1}^{2}}{2 m^{*}}$. An exponentially decaying cosine

$$
s_{\omega_{1}}^{\prime x}(t) \approx s_{\omega_{1}}^{\prime x}(0) e^{-t / \tau_{\perp}} \cos \left(\omega_{M}^{\prime} t\right)
$$

is fit to the non-Markovian spin dynamics in order to find a value for the effective decay rate $\tau_{\perp}^{-1}\left(\omega_{1}\right)$ and the precession frequency $\omega_{M}^{\prime}\left(\omega_{1}\right)$. The relative renormalization of the precession frequency is given by $\frac{\Delta \omega}{\omega_{M}}$ with $\Delta \omega=\omega_{M}^{\prime}-\omega_{M}$.

Fig. 1 shows the relative frequency renormalization obtained from a fit to the non-Markovian calculation and the corresponding Markovian result for a $\delta$-like initial spectral electron distribution as a function of the kinetic energy $\hbar \omega_{1}$. First of all, it can be seen that in threedimensional as well as in two-dimensional systems the Markovian and non-Markovian results coincide. In the three-dimensional case, the square-root energy dependence of the renormalization for $\omega_{1}<\omega_{M}$ can be seen 


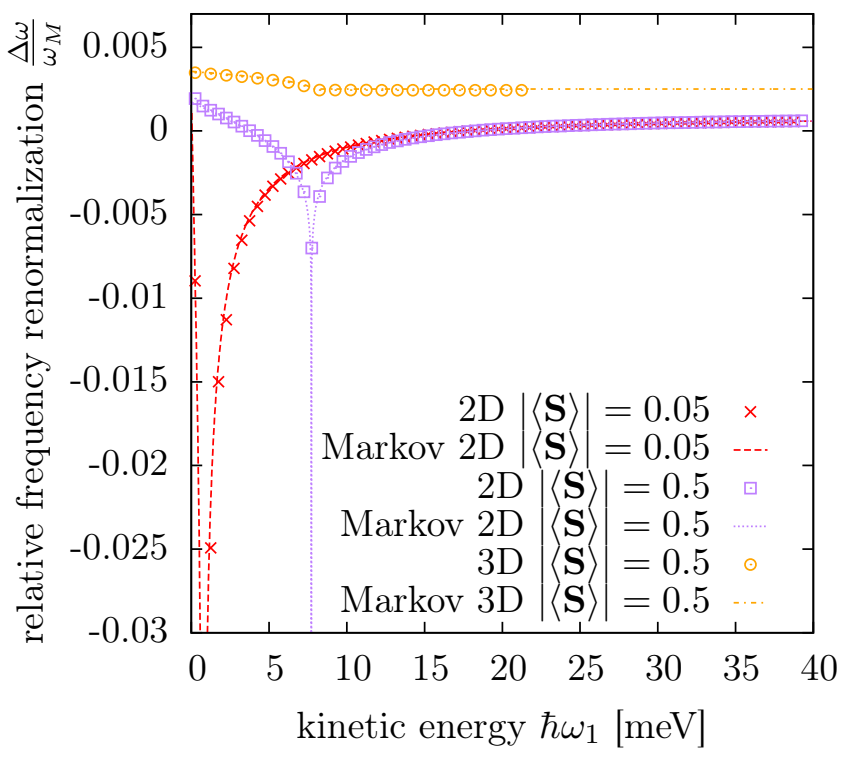

FIG. 1. Dependence of the relative frequency renormalization $\frac{\Delta \omega}{\omega_{m}}$ on the kinetic electron energy for two- and threedimensional systems according to the calculation including a finite memory [Eqs. (15)] (points) and in the Markov limit [Eqs. (10)] (lines) for different values of the average impurity spin $|\langle\mathbf{S}\rangle|$ (in units of $\hbar$ ).

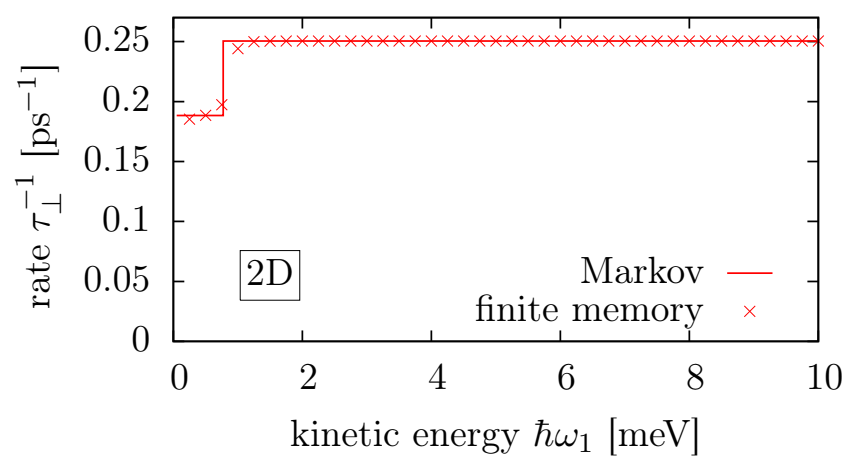

FIG. 2. Spin transfer rate $\tau_{\perp}^{-1}$ in Markov approximation (line) according to Eq. (8) and exponential fit to the calculation including a finite memory (crosses) using Eq. (15) for $|\langle\mathbf{S}\rangle|=$ 0.05 in a two-dimensional system.

clearly, while in two dimensions, the logarithmic divergence at $\omega_{1}=\omega_{M}$ is apparent. The positive relative frequency renormalization in three dimensions describes an increase in the modulus of the precession frequency. In two dimensions, the slightly positive background of the renormalization is overcompensated by a negative value in the region around the divergence.

In Fig. 2, the spin transfer rate according to the Markov approximation is compared with the value obtained by the exponential fit to the non-Markovian result for a calculation with $|\langle\mathbf{S}\rangle|=0.05$ in two dimensions. The step in the rate $\tau_{\perp}^{-1}$ at $\omega_{1}=\omega_{M}$, which is predicted in the Markov limit [cf. Eq. (8)], is found to be slightly rounded off in the non-Markovian calculation, but the deviations

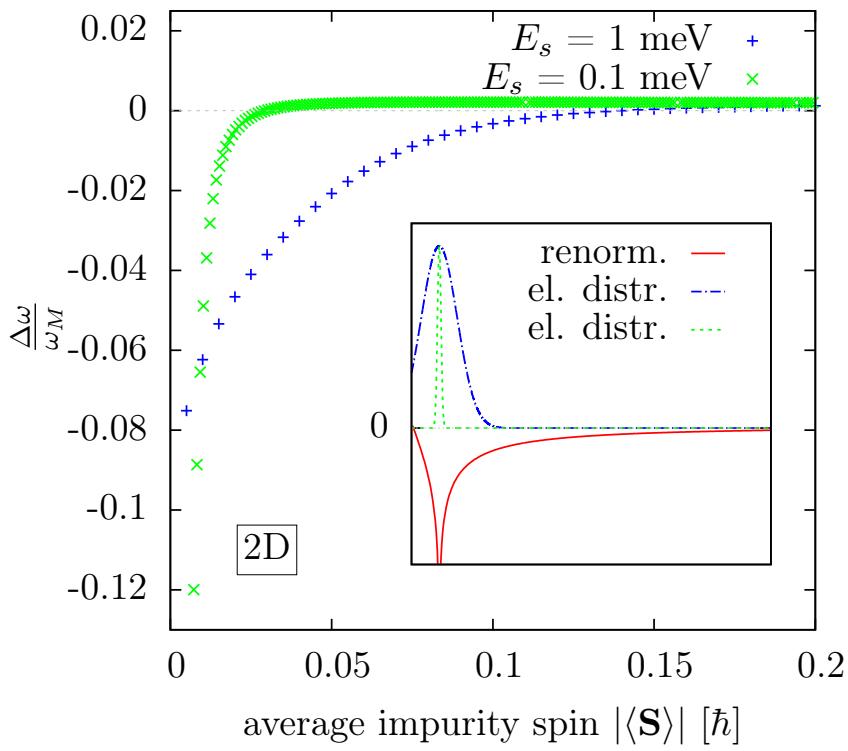

FIG. 3. Relative frequency renormalization $\frac{\Delta \omega}{\omega_{M}}$ in a twodimensional system for a Gaussian spectral electron distribution centered at $E_{c}=\hbar \omega_{M}$ with standard deviations of $E_{s}=1$ $\mathrm{meV}$ and $E_{s}=0.1 \mathrm{meV}$, respectively. The initial electron distribution as a function of the kinetic energy is visualized in the inset as the blue dash-dotted line $\left(E_{s}=1 \mathrm{meV}\right)$ and green dotted line $\left(E_{s}=0.1 \mathrm{meV}\right)$ together with the corresponding frequency renormalization for $\delta$-like excitations (red line) for $|\langle\mathbf{S}\rangle|=0.05$.

between both results are rather small.

In order to find an estimate for the strength of the change of the precession frequency for a more realistic electron distribution, Fig. 3 shows the relative precession frequency renormalization as a function of the average impurity spin where the initial spectral electron distribution [cf. inset of Fig. 3] was assumed to be Gaussian with center at $E_{c}=\hbar \omega_{M}$ and standard deviation $E_{s}=1$ $\mathrm{meV}(0.1 \mathrm{meV})$ corresponding to a full width at half maximum $(\mathrm{FWHM})$ of $\approx 2.35 \mathrm{meV}(0.235 \mathrm{meV})$ or a Gaussian envelope of an exciting laser pulse with a duration (FWHM) of $\approx 140 \mathrm{fs}$ (1.4 ps). The calculations for Fig. 3 were performed using the 2D Markovian expression for the rates in Eq. (8) and the renormalized precession frequencies in Eq. (10a). It can be seen that the magnitude of the frequency renormalization can reach values of several percent of the mean-field precession frequency and is negative for small values of $|\langle\mathbf{S}\rangle|$. For larger values of the impurity magnetization, the frequency renormalization approaches a small positive value. One could expect that the narrower electron distribution $\left(E_{s}=0.1 \mathrm{meV}\right)$ is closer to the $\delta$-like case than the wider distribution $\left(E_{s}=1 \mathrm{meV}\right)$ and therefore the frequency renormalization should be more pronounced. However, it can be seen from Fig. 3 that this is only the case for very low values of $|\langle\mathbf{S}\rangle|$ (below 0.01 in the case studied here). For higher values of the impurity magnetization, the relative frequency renormalization approaches the positive back- 


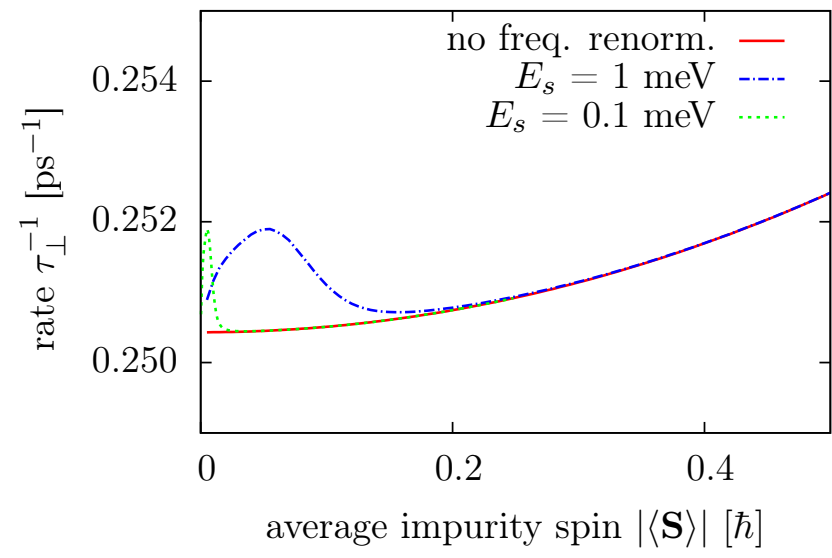

FIG. 4. Rate $\tau_{\perp}^{-1}$ obtained from an exponential fit for Gaussian initial spectral electron distributions (cf. Fig. 3) as a function of the average impurity spin with (blue dash-dotted line/green dotted line) and without (red solid line) accounting for a renormalization of the precession frequency.

ground much faster in the calculations with the narrower electron distribution.

Note that in order to be able to measure or fit a precession frequency, at least one period of the oscillations should be visible before the spin polarization is decayed. Thus, the minimal value of the impurity magnetization, where one can reasonable deduce a precession frequency from the time evolution of the spin polarization, is given by $\left|\omega_{M}\right| \gtrsim \tau_{\perp}^{-1}$ which yields, for the parameters above, $|\langle\mathbf{S}\rangle| \gtrsim 0.01$. Therefore, we find that short laser pulses with pulse durations of the order of 100 fs provide the most promising configuration for experiments to measure the frequency renormalization.

Since the frequency renormalization depends on the kinetic energy and therefore the wave-vector of an electron, the question arises, whether this dependence leads to a dephasing of spins of electrons with different $\mathbf{k}$-vectors. To address this question, we show in Fig. 4 the value of the rate $\tau_{\perp}^{-1}$ obtained by an exponential fit to the time evolution of the total carrier spin polarization, where the same Gaussian initial electron distributions are used as in Fig. 3. It can be seen that calculations, where the correlation induced frequency renormalization is neglected, produce very similar decay rates as calculations that account for this renormalization for most of the possible values of the impurity magnetization. Only in a regime where the impurity spin is small we find a slightly larger value $(\lesssim 1 \%)$ of the rate at $|\langle\mathbf{S}\rangle| \approx 0.05$ for $E_{s}=1 \mathrm{meV}$ and $|\langle\mathbf{S}\rangle| \approx 0.005$ for $E_{s}=0.1 \mathrm{meV}$. This increasing decay is the consequence of the dephasing of electron spins due to the $\mathbf{k}$-dependence of the frequency renormalization. Since the expression for the rate in the Markov limit [cf. Eq. (8)] and the frequency renormalization [cf. Eq. (10a)] depend on the same parameters, this dephasing mechanism is always accompanied by a genuine spin transfer between impurities and carriers that is typically much faster than the dephasing itself.

\section{CORRELATION ENERGY}

Most studies on DMS which probe the energies of electrons in DMS use the mean-field approximation ${ }^{24}$ and describe the effects of the impurity magnetization as a renormalization of the electron g-factor which is known as the giant Zeeman effect ${ }^{56}$. If, however, the build-up of carrier-impurity correlations is taken into account, the mean $s$ - $d$ exchange interaction energy $\left\langle H_{s d}\right\rangle$ will deviate from the mean-field value. The correlation energy can, in principle, have an impact on the thermodynamic properties of DMS which could help, e. g., in the description of the paramagnetic-ferromagnetic phase transition in GaMnAs.

Since the derivation of the PESC-equations in Ref. 49 required finding explicit expressions for the correlations, we can use this theory to get the correlation induced correction $\left\langle H_{s d}^{c o r}\right\rangle$ to the mean-field exchange interaction energy analytically:

$$
\begin{aligned}
\left\langle H_{s d}\right\rangle & =\frac{J_{s d}}{V} \sum_{\substack{I n n^{\prime} \\
\sigma \sigma^{\prime} \mathbf{k} \mathbf{k}^{\prime}}} \mathbf{S}_{n n^{\prime}} \mathbf{S}_{\sigma \sigma^{\prime}}\left\langle c_{\sigma \mathbf{k}}^{\dagger} c_{\sigma^{\prime} \mathbf{k}^{\prime}} e^{i\left(\mathbf{k}^{\prime}-\mathbf{k}\right) \mathbf{R}_{I}} \hat{P}_{n n^{\prime}}^{I}\right\rangle= \\
& =: \sum_{\mathbf{k}} \hbar \boldsymbol{\omega}_{M} \cdot \mathbf{s}_{\mathbf{k}}+\left\langle H_{s d}^{c o r}\right\rangle
\end{aligned}
$$

Using the time-integral form of the correlations from Ref. 49 we find:

$$
\begin{aligned}
\left\langle H_{s d}^{c o r}\right\rangle & =\frac{J_{s d}}{V} n_{M n} \sum_{\mathbf{k k}^{\prime}} \sum_{\alpha=1}^{3} Q_{\alpha \mathbf{k}}^{\alpha \mathbf{k}^{\prime}}=-\hbar \sum_{\mathbf{k}_{1} \mathbf{k}_{2}}\left\{\operatorname{Im}\left\{G_{\omega_{\mathbf{k}_{2}}}^{\omega_{\mathbf{k}_{1}}+\omega_{M}}\right\}\left[\frac{b^{+}}{2} n_{\mathbf{k}_{2}}^{\downarrow}-\frac{b^{-}}{2} n_{\mathbf{k}_{1}}^{\uparrow}-\frac{b^{0}}{2}\left(n_{\mathbf{k}_{1}}^{\uparrow} n_{\mathbf{k}_{2}}^{\downarrow}+n_{\mathbf{k}_{2}}^{\uparrow} n_{\mathbf{k}_{1}}^{\downarrow}\right)\right]+\right. \\
& \left.+\operatorname{Im}\left\{G_{\omega_{\mathbf{k}_{2}}}^{\omega_{\mathbf{k}_{1}}-\omega_{M}}\right\}\left[\frac{b^{-}}{2} n_{\mathbf{k}_{2}}^{\uparrow}-\frac{b^{+}}{2} n_{\mathbf{k}_{1}}^{\downarrow}+\frac{b^{0}}{2}\left(n_{\mathbf{k}_{1}}^{\uparrow} n_{\mathbf{k}_{2}}^{\downarrow}+n_{\mathbf{k}_{2}}^{\uparrow} n_{\mathbf{k}_{1}}^{\downarrow}\right)\right]+\operatorname{Im}\left\{G_{\omega_{\mathbf{k}_{2}}}^{\omega_{\mathbf{k}_{1}}}\right\}\left[\frac{b^{\|}}{4}\left(\left(n_{\mathbf{k}_{2}}^{\uparrow}+n_{\mathbf{k}_{2}}^{\downarrow}\right)-\left(n_{\mathbf{k}_{1}}^{\uparrow}+n_{\mathbf{k}_{1}}^{\downarrow}\right)\right)\right]\right\}
\end{aligned}
$$

To understand Eq. (18) it is important to recall that the correlations typically build up on the timescale of a few 
$\mathrm{fs}^{52}$, while the spin-up and spin-down occupations change on a ps timescale ${ }^{46,49}$. Thus, $\left\langle H_{s d}^{c o r}\right\rangle$ can be interpreted as a quasi-equilibrium value of the correlation energy for given values of adiabatically changing occupations $n_{\mathbf{k}_{1}}^{\uparrow / \downarrow}$.

As in the discussion of the frequency renormalization, we neglect terms of second order in the electron variables and apply the Markov approximation to find for the twodimensional case:

$$
\begin{aligned}
\left\langle H_{s d}^{c o r}\right\rangle \approx & -\frac{J_{s d}^{2}}{\hbar} \frac{n_{M n}}{V} \frac{A m^{*}}{2 \pi \hbar} \sum_{\mathbf{k}_{1}} \\
& \left\{\ln \left|\frac{\omega_{B Z}-\left(\omega_{\mathbf{k}_{1}}+\omega_{M}\right)}{\omega_{\mathbf{k}_{1}}+\omega_{M}}\right| b^{-} n_{\mathbf{k}_{1}}^{\uparrow}+\right. \\
& +\ln \left|\frac{\omega_{B Z}-\left(\omega_{\mathbf{k}_{1}}-\omega_{M}\right)}{\omega_{\mathbf{k}_{1}}-\omega_{M}}\right| b^{+} n_{\mathbf{k}_{1}}^{\downarrow}+ \\
& \left.+\ln \left|\frac{\omega_{B Z}-\omega_{\mathbf{k}_{1}}}{\omega_{\mathbf{k}_{1}}}\right| \frac{b^{\|}}{2}\left(n_{\mathbf{k}_{1}}^{\uparrow}+n_{\mathbf{k}_{1}}^{\downarrow}\right)\right\}
\end{aligned}
$$

The mathematical structure of the correlation energy $\left\langle H_{s d}^{c o r}\right\rangle$ in Eq. (19) is very similar to that of the frequency renormalization in Eq. (9). To see this relation, it is helpful to express the occupations $n_{\mathbf{k}_{1}}^{\uparrow / \downarrow}$ of the spin-up and spin-down subbands in terms of the occupation $n_{\mathbf{k}_{1}}$ of both bands and the spin component $s_{\mathbf{k}_{1}}^{\|}$parallel to the impurity magnetization (quantization axis) via

$$
n_{\mathbf{k}_{1}}^{\uparrow / \downarrow}=\frac{n_{\mathbf{k}_{1}}}{2} \pm s_{\mathbf{k}_{1}}^{\|} .
$$

As it is common for spin-dependend single particle energies like the Dresselhaus- ${ }^{57}$ or Rashba-terms ${ }^{58}$, one could expect that the spin-dependend part of the correlation energy can be written as an effective magnetic field in which the electron spins precess. This additional precession movement could be made responsible for the frequency renormalization discussed above. However, although the corresponding effective field due to the correlation energy has the same form as the frequency renormalization, it is larger by a factor of 2 . We attribute this to the fact that the correlation energy is not an average over a Hermitian single particle operator, but comprises multiparticle effects, where the naive identification of an effective magnetic field can lead to incorrect predictions.

A particularly interesting and transparent case is that where the impurity magentization $\langle\mathbf{S}\rangle$ vanishes. Then, the correlation energy takes the form

$$
\left\langle H_{s d}^{c o r}\right\rangle=-\frac{J_{s d}^{2}}{\hbar} \frac{n_{M n}}{V}\left\langle S^{2}\right\rangle \frac{A m^{*}}{2 \pi \hbar} \sum_{\mathbf{k}_{1}} \ln \left|\frac{\omega_{B Z}-\omega_{\mathbf{k}_{1}}}{\omega_{\mathbf{k}_{1}}}\right| n_{\mathbf{k}_{1}}
$$

Thus, for $\langle\mathbf{S}\rangle=0$, we find a logarithmic divergence of the correlation energy with respect to $\omega_{B Z} \rightarrow \infty$ and $\omega_{\mathbf{k}_{1}} \rightarrow 0$. In both limits, the correlation energy is negative and independent of the sign of the coupling constant $J_{s d}$. The negative correlation energy suggests a formation of correlated carrier-impurity states, i. e., magnetic polarons. Such states have also been predicted in $\mathrm{GaMnAs}^{39}$, where the correlation energy stems from an attractive Coulomb potential due to the charged state of the Mn ions which acts, e. g., as acceptors, if they substitute $\mathrm{Ga}$ atoms in the GaAs crystal lattice. An additional contribution to the correlation energy originating from the spin-dependent $s$ - $d$ interaction could enhance the formation of the magnetic polarons in GaMnAs. Furthermore, our finding is consistent with the fact that also in the solution of the Kondo problem, the ground state is strongly correlated ${ }^{2}$.

Again, the divergence at $\omega_{\mathbf{k}_{1}} \rightarrow 0$ in Eq. (21) is integrable so that the total correlation energy always assumes finite values. To estimate the magnitude of the correlation energy we consider the case where $\langle\mathbf{S}\rangle=0$ and the spectral electron distribution is given by a Gaussian centered at the band edge with standard deviation $E_{s}=1 \mathrm{meV}$. For the parameters of the $\mathrm{Cd}_{0.93} \mathrm{Mn}_{0.07} \mathrm{Te}$ quantum well discussed above, we find from Eq. (21) a value of $\left\langle H_{s d}^{c o r}\right\rangle \approx-1.8 \mu \mathrm{eV}$ per electron. Thus, the correlations can be destroyed by thermal fluctuations when the temperature $T$ exceeds $20 \mathrm{mK}$.

\section{CONCLUSION}

A microscopic quantum kinetic theory is employed to describe the spin dynamics of carriers and magnetic impurities in diluted magnetic semiconductors (DMS) accounting also for the dynamics of the carrier-impurity correlations. The role of the correlations is examined to shed light into the controversy about their importance: While some authors assume that the Kondo physics due to carrier-impurity correlation is of minor importance ${ }^{37}$, others $^{38}$ find divergences in a perturbative treatment of the spin dynamics in DMS, similarly to the appearance of divergences found in the metallic Kondo-effect ${ }^{1}$. In the present study, we find that the correlations, besides mediating the spin transfer between carriers and impurities, are also responsible for a renormalization of the precession frequency of up to a few percent in two-dimensional structures. Since for these values, the relative frequency renormalization is negative, the precession frequency of the electron spin is reduced.

In order to experimentally probe the correlation induced frequency renormalization, the spectral features of the laser pulse have to be precisely controlled. Furthermore, it was reported ${ }^{59}$ that an antiferromagnetic impurity-impurity interaction influences the thermal equilibrium value of the Mn magnetization, which in turn changes the measured electron spin precession frequency. Therefore, it is common to introduce a fitting parameter $T_{0}$ and describe the equilibrium Mn magnetization by a Brillouin function with effective temperature $T_{\text {eff }}=T_{0}+T$, where $T$ is the temperature of the sample. This complicates the identification of correlation induced changes in the precession frequency. To distinguish both 
effects it is useful that in addition to the dependence on the spectral position and shape of the exciting pulse, the relative frequency renormalization due to the correlations is independent of the impurity density, while the impurity-impurity interaction depends on the mean distance between the impurity ions and is not influenced by the excitation conditions. Because of this and from the different parameters entering the prefactor of the frequency renormalization, we find that the most promising samples for experimentally accessing the correlation induced frequency renormalization are very narrow quantum wells with large effective masses and a large coupling constant $J_{s d}$ while the impurity concentration should be relatively low. Also, we find that the spectral properties of ultrashort pulses with durations in the $100 \mathrm{fs}$ range suit this purpose.

Although the $\mathbf{k}$-dependence of the frequency renormalization can in principle lead to a dephasing of carrier spins, the spin transfer from the carriers to the impurities is usually much faster, so that this dephasing mechanism yields only very small corrections to the total decay of the carrier spin.

By comparing the results of the quantum kinetic dynamics including the time evolution of correlations with the Markov limit, where the correlations can be expressed in terms of carrier and impurity variables alone, we find that simple Markovian expressions for spin transfer rate and frequency renormalization reproduce the results of the quantum kinetic treatment very well. Although logarithmic divergences are found for some values of carrier wave-vectors as well as for bandwidth $\rightarrow \infty$, similar to the metallic Kondo effect, the total frequency renormalization, which is obtained by integrating with a smooth spectral carrier-distribution, is always finite. Also, the divergences only appear in the Markov limit, while the effects induced by the correlations are analytic by construction when a finite memory is accounted for.

The explicit expressions for the correlations in the Markov limit also allow to find an equilibrium value for the correlation energy in terms of carrier and impurity variables. The form of the correlation energy is similar to the expression for the frequency renormalization and hints towards the appearance of correlated carrierimpurity states for low temperatures, independent of the sign of the coupling constant. However, the required temperatures are lower than the values that are typically considered in experimental setups probing DMS. Thus, in these cases, the correlation energy is not expected to influence the thermodynamic properties of DMS strongly.

Although many similarities between the DMS systems and the Kondo problem can be found, a one-to-one correspondence can not be established since the density of states in semiconductors is much smaller than in metals. Hence, the assumption that the number of magnetic impurities exceed the number of quasi-free carriers, that is valid for DMS and has been used in the derivation of the equations of motion on which the present study is based ${ }^{49}$, is opposite to the situation in usual metallic Kondo-systems. Furthermore, the large number of carriers in the metal can be expected to lead to a fast carrier spin relaxation due to Coulomb scattering. However, the approach of finding a quasi-equilibrium value for the correlation energy from a quantum kinetic theory could be extended and might help to understand the Kondo physics from a new point of view. Since we find that the correlation effects on the spin dynamics and the correlation energies are linear in the density of states, our theory indeed agrees with the finding of strongly correlated states for low temperatures in metallic Kondo systems.

\section{ACKNOWLEDGMENTS}

We gratefully acknowledge the financial support of the Deutsche Forschungsgemeinschaft through grant No. AX17/9-1, from the Universidad de Buenos Aires, project UBACyT 2011-2014 No. 20020100100741, and from CONICET, project PIP 11220110100091.
1 J. Kondo, Progress of Theoretical Physics 32, 37 (1964).

2 A. C. Hewson, The Kondo Problem to Heavy Fermions (Cambridge University Press, 1993).

3 S. M. Cronenwett, T. H. Oosterkamp, and L. P. Kouwenhoven, Science 281, 540 (1998).

4 T. Inoshita, Science 281, 526 (1998).

5 A. Kaminski, Y. V. Nazarov, and L. I. Glazman, Phys. Rev. Lett. 83, 384 (1999).

${ }^{6}$ W. G. van der Wiel, S. D. Franceschi, T. Fujisawa, J. M. Elzerman, S. Tarucha, and L. P. Kouwenhoven, Science 289, 2105 (2000).

7 J. Elzerman, S. De Franceschi, D. Goldhaber-Gordon, W. van der Wiel, and L. Kouwenhoven, Journal of Low Temperature Physics 118, 375 (2000).

8 P. Nordlander, N. S. Wingreen, Y. Meir, and D. C. Langreth, Phys. Rev. B 61, 2146 (2000).

9 H. Jeong, A. M. Chang, and M. R. Melloch, Science 293,
2221 (2001).

10 D. Goldhaber-Gordon, H. Shtrikman, D. Mahalu, D. Abusch-Magder, U. Meirav, and M. A. Kastner, Nature 391, 156 (1998).

11 A. Micolich, Nature Physics 9, 530531 (2013).

12 B. Hemingway, S. Herbert, M. Melloch, and A. Kogan, Phys. Rev. B 90, 125151 (2014).

13 T. Dietl and H. Ohno, Rev. Mod. Phys. 86, 187 (2014).

14 M. W. Wu, J. H. Jiang, and M. Q. Weng, Phys. Rep. 493, 61 (2010).

15 L. Cywiński and L. J. Sham, Phys. Rev. B 76, 045205 (2007).

16 M. Nawrocki, R. Planel, G. Fishman, and R. Galazka, Phys. Rev. Lett. 46, 735 (1981).

17 S. A. Crooker, D. D. Awschalom, J. J. Baumberg, F. Flack, and N. Samarth, Phys. Rev. B 56, 7574 (1997).

18 J. H. Jiang, Y. Zhou, T. Korn, C. Schüller, and M. W. 
Wu, Phys. Rev. B 79, 155201 (2009).

19 T. Dietl, Nat. Mater. 9, 965974 (2010).

20 D. Awschalom and M. Flatté, Nature Physics 3, 153 (2007).

${ }^{21}$ K. Edmonds, G. van der Laan, and G. Panaccione, Semicond. Sci. Technol. 30, 043001 (2015).

${ }^{22}$ M. D. Kapetanakis, J. Wang, and I. E. Perakis, J. Opt. Soc. Am. B 29, A95 (2012).

23 J. Kossut, physica status solidi (b) 72, 359 (1975).

24 J. K. Furdyna, J. Appl. Phys. 64, R29 (1988).

25 H. Krenn, K. Kaltenegger, T. Dietl, J. Spałek, and G. Bauer, Phys. Rev. B 39, 10918 (1989).

26 J. M. Kikkawa and D. D. Awschalom, Phys. Rev. Lett. 80, 4313 (1998).

27 A. V. Akimov, A. V. Scherbakov, D. R. Yakovlev, I. A. Merkulov, M. Bayer, A. Waag, and L. W. Molenkamp, Phys. Rev. B 73, 165328 (2006).

${ }^{28}$ K. E. Rönnburg, E. Mohler, H. G. Roskos, K. Ortner, C. R. Becker, and L. W. Molenkamp, Phys. Rev. Lett. 96, 117203 (2006).

${ }^{29}$ M. D. Kapetanakis, I. E. Perakis, K. J. Wickey, C. Piermarocchi, and J. Wang, Phys. Rev. Lett. 103, 047404 (2009).

30 J. Kossut and J. Gaj, eds., Introduction to the Physics of Diluted Magnetic Semiconductors, Springer Series in Materials Science No. 144 (Springer, Berlin, 2011).

31 C. Camilleri, F. Teppe, D. Scalbert, Y. Semenov, M. Nawrocki, M. Dyakonov, J. Cibert, S. Tatarenko, and T. Wojtowicz, Phys. Rev. B 64, 085331 (2001).

32 R. C. Myers, M. H. Mikkelsen, J.-M. Tang, A. C. Gossard, M. E. Flatté, and D. D. Awschalom, Nature Materials 7, 203 (2008).

33 S. Cronenberger, M. Vladimirova, S. Andreev, M. Lifshits, and D. Scalbert, Phys. Rev. Lett. 110, 077403 (2013).

34 T. Dietl, H. Ohno, F. Matsukura, J. Cibert, and D. Ferrand, Science 287, 1019 (2000).

35 H. Ohno, Nat. Mater. 9, 952 (2010).

36 S. A. Wolf, D. D. Awschalom, R. A. Buhrman, J. M. Daughton, S. von Molnr, M. L. Roukes, A. Y. Chtchelkanova, and D. M. Treger, Science 294, 1488 (2001).

37 S. Das Sarma, E. H. Hwang, and A. Kaminski, Phys. Rev. B 67, 155201 (2003).

38 O. Morandi and P.-A. Hervieux, Phys. Rev. B 81, 195215 (2010).

39 H. Bednarski and J. Spałek, Journal of Physics: Condensed
Matter 24, 235801 (2012).

${ }^{40}$ Y. Tian, Y. Li, M. He, I. A. Putra, H. Peng, B. Yao, S. A. Cheong, and T. Wu, Appl. Phys. Lett. 98, 162503 (2011).

41 D. E. Angelescu and R. N. Bhatt, Phys. Rev. B 65, 075211 (2002).

42 A. C. Durst, R. N. Bhatt, and P. A. Wolff, Phys. Rev. B 65, 235205 (2002).

43 M. Herbich, A. Twardowski, D. Scalbert, and A. Petrou, Phys. Rev. B 58, 7024 (1998).

44 P. A. Wolff, R. N. Bhatt, and A. C. Durst, J. Appl. Phys. 79, 5196 (1996).

45 C. Thurn and V. M. Axt, Phys. Rev. B 85, 165203 (2012).

46 C. Thurn, M. Cygorek, V. M. Axt, and T. Kuhn, Phys. Rev. B 87, 205301 (2013).

47 C. Thurn, M. Cygorek, V. M. Axt, and T. Kuhn, Phys. Rev. B 88, 161302(R) (2013).

48 M. Cygorek and V. M. Axt, Phys. Rev. B 90, 035206 (2014).

49 M. Cygorek and V. M. Axt, Semicond. Sci. Technol. 30, 085011 (2015).

50 F. Ungar, M. Cygorek, P. I. Tamborenea, and V. M. Axt, Phys. Rev. B 91, 195201 (2015).

51 In order to obtain physically meaningful results, care has to be taken that the mean-field exchange energy between carriers and impurites is taken into account in the corresponding $\delta$-function in the expression for Fermi's golden rule $^{49}$.

52 M. Cygorek and V. M. Axt, "Non-markovian effects in the spin transfer dynamics in diluted magnetic semiconductors due to excitation in proximity to the band edge," (2015), accepted in JPCS.

53 S. Doniach and E. H. Sondheimer, Green's Functions for Solid State Physicists (Imperial College Press, 1998).

${ }^{54}$ K. G. Wilson, Rev. Mod. Phys. 47, 773840 (1975).

55 P. Y. Yu and M. Cardona, Fundamentals of Semiconductors, 4th ed. (Springer, Heidelberg, 2010).

56 S. A. Crooker, E. Johnston-Halperin, D. D. Awschalom, R. Knobel, and N. Samarth, Phys. Rev. B 61, R16307 (2000).

57 G. Dresselhaus, Phys. Rev. 100, 580 (1955).

${ }^{58}$ Y. A. Bychkov and E. I. Rashba, Journal of Physics C: Solid State Physics 17, 6039 (1984).

59 J. Gaj, R. Planel, and G. Fishman, Solid State Commun. 29, 435 (1979). 